[15]

Knapp, C., and Carter, G.

The generalized correlation method for estimation of time delay.

IEEE Transactions on Acoustics, Speech, and Signal Processing, ASSP-24, 4 (Aug. 1976).

[16] Eid, E., Chan, T., Fossum, E., Tsai, R., Spagnuolo, R., Deily, J., Byers, W., and Peden, J.

Design and characterization of ionizing radiation-tolerant CMOS APS image sensors up to $30 \mathrm{Mrd}$ ( $\mathrm{Si}$ ) total dose. IEEE Transactions on Nuclear Science, 48, 6 (Dec. 2001).

[17] Duhamel, P.

Implementation of "split-radix" FFT algorithms for complex, real, and real-symmetric data. IEEE Transactions on Acoustic, Speech, and Signal Processing, 34, 2 (1986).

[18] Rajan, S., Wang, S., Inkol, R., and Joyal, A. Efficient approximations for the arctangent function. IEEE Signal Processing Magazine, (May 2006), 108-111.

[19] Cormen, T, Leiserson, C., and Rivest, R.

Introduction to Algorithms.

Cambridge, MA: MIT Press, 1990, 769-771.

\section{Discriminating Complex Bodies with Minor Variations using E-Pulse}

In this paper, we present a technique to discriminate between two complex bodies with minor structural variations using the high frequency poles as the discriminating feature in the extinction-pulse (E-pulse) scheme. A full scale model of MiG-29 aircraft is considered for discrimination against another with stores (missiles) attached below the wings. The MiG-29 aircraft is impinged with a radar impulse to excite the electrical natural resonance frequencies of the aircraft. These natural resonance frequencies are unique to any aircraft and are used as distinct features for discriminating it against another MiG-29 aircraft with stores. Prony's method is used to extract the natural frequencies from the impulse response of the aircraft.

\section{INTRODUCTION}

Discrimination of complex targets such as aircraft is vital for timely and corrective action to respond to unidentified targets in a real scenario. Incorrect recognition of target type can lead to disasters [1]. It is therefore essential to evolve techniques to discriminate aircraft of different classes. Due to the inherent complexity of the aircraft surface geometry, it

Manuscript received November 11, 2003; revised April 4, 2007; released for publication November 8, 2007.

IEEE Log No. T-AES/44/1/920416.

Refereeing of this contribution was handled by P. K. Willett.

0018-9251/08/\$25.00 (C) 2008 IEEE is, in general, difficult to formulate and evolve such a technique using a full-scale model. Accordingly, simplified models that preserve global features are used in order to make it mathematically tractable.

Boeing 727 and 737 aircraft were discriminated in [2] over a range of aspect angles using high resolution range profiles (HRRP) and generalized linear discriminant. Using HRRP, a study on automatic target recognition for moving targets is presented in [3]. However, HRRP requires radar echoes from more than one aspect angle to ascertain discrimination. In this paper, an alternative aspect-independent natural resonance based technique is presented.

Target recognition using complex natural resonances (electrical) was first introduced in [4]. It was established that 1) only a few natural resonances of a body are adequate to discriminate it from a finite collection of bodies, and 2) the natural resonances of a body are independent of aspect angle. Based on this, several target discrimination methods have been proposed. In one class of methods, the estimated natural resonances of an unknown target is compared with a library of natural resonances of known targets $[4,5]$. In the second class of techniques, the natural frequencies of known targets are used to synthesize discriminant signals [6]. These signals when subsequently convolved with the late-time target response leads to an expected response. If the unknown target has a different geometry or composition, the signal resulting from this convolution will be different from the expected one.

One such scheme belonging to the second class of techniques is based on extinction-pulse (E-pulse) and has been used extensively for discrimination between targets of different classes [6]. An E-pulse is a transient finite duration waveform which annihilates the contribution of a select number of the natural resonances (i.e., poles) in the late-time response [6]. (Essentially, an E-pulse places zeros at the same location as the natural resonances thereby annihilating their contribution in the target's impulse response. Equivalently, with respect to a suitable basis functions, the E-pulse belongs to the null space of a matrix related to the natural resonances of the target.) Since the values of the target resonance frequencies are, in general, independent of the excitation waveform, the E-pulse will eliminate the desired natural modal content of the late-time scattered field regardless of the orientation of the target with respect to the transmitting and receiving antennas. Each target is characterized by a unique set of natural frequencies. An E-pulse designed to annul certain natural resonances of one target will excite those of a target with different natural frequencies, resulting in a different scattered field [6].

The discrimination technique involves generation of E-pulses which are unique for each target and stored in a library. During the discrimination process, 
an impulse (or near impulse) waveform is transmitted and the response convolved with all the E-pulses stored in the library. The convolved output is checked for zero (near zero) signal in the late time. The unknown target is then declared consistent with the target whose E-pulse gave zero signal in the convolved output. Thus, the target is discriminated against the other targets whose E-pulses are generated and stored in the library.

Lin and Coradaro [7] have modeled the aircraft as thin wire structure with swept wings and computed the surface currents using the thin wire time-domain computer code. The surface current was computed for each segment on the wire and the natural frequencies were extracted using the Prony's method. Mooney, Ding and Riggs [8] have developed a generalized likelihood ratio test (GLRT) which is superior to E-pulse scheme as regards the performance in the presence of noise is concerned. They have demonstrated the target discrimination capability by using the GLRT technique on four types of general class of aircraft modeled as thin wire stick models. Rothwell et al. [6] have demonstrated the success of E-pulse technique for target discrimination using measured response from scaled models of Boeing 707 and McDonell Douglas F-18 aircraft. Li et al. [9] have used the combination of early/late time E-pulse technique for target identification. They have used the examples of scaled models of B-52, B-58, and F-14 aircraft. Their method reduces the early-time processing effort by narrowing down the number of potential targets, and by determining the most likely range of angles for each target. Baum et al. [10] have described the methodology adopted for accurately discriminating scaled models of eight aircraft targets (B-1 Bomber, small F-15, tiny 747, medium 707, big F-15, F-18, small 707, and A-10) among each other. The targets included simple aluminium models as well as detailed cast-metal models ranging in fuselage length from 6 in to 18 in. For all the above cases, effective discrimination has been shown using the E-pulse scheme. The aspect independence of the natural frequencies and the generalization property of an artificial neural network have been efficiently combined to yield high classification rates in a noise environment in [11]; however, the measured data used have been obtained from B2 and F14 scaled models.

Evidently, most of the work described earlier involves the use of scaled or stick model. It is, however, not clear whether or not the natural frequencies derived from such models are directly scalable to those derived from full-scale models of complex objects such as MiG-29. Therefore, it is expected that using full-scale models would yield better results when targets with minor variations in structural or material composition are to be discriminated. We present a technique here using the E-pulse scheme using higher order natural resonances for the discrimination of a MIG-29 aircraft against another carrying stores, thereby demonstrating its capability to discriminate targets with minor structural variations. Albeit only low-order natural resonances are needed to discriminate aircraft of different types, higher order modes are required to discriminate an aircraft and its minor variant. The fact that the performance of the radar target recognition scheme improves with an increase in the number of the natural frequencies and with an increase in the signal-to-noise ratio has been shown, and a closed-form expression for the normalized estimation error has been derived in [12].

Section II describes the methodology used to discriminate between targets with minor variations. It also describes the E-pulse discrimination scheme and defines a performance measure to indicate the improvement in the discrimination procedure by using high frequency poles. Section III describes the aircraft modeling and discrimination effectiveness with higher frequency poles included in the E-pulse generation. Section IV gives an account of the conclusions drawn from the specific example of discrimination between a basic aircraft and its variant.

\section{FORMULATION OF THE PROBLEM}

In this section, a full-scale model of an MiG-29 aircraft is obtained using canonical shapes and discriminated against another MiG-29 aircraft with stores loaded under the wings. An examination of electromagnetic response of a target of lateral dimension (maximum dimension along either axis) $l$ indicates that the natural frequency range corresponding to $l / 2 \leq \lambda \leq 10 l$ provides the most useful initial information [13]. While the higher frequencies characterize the finer details of the object, the lower frequency provide information such as overall dimension, approximate shape and material decomposition [13]. Therefore, in this work, higher frequency natural resonances are also used to generate E-pulses.

The proposed scheme involves the following steps. 1) Geometrically model the targets to be discriminated; 2) extract the complex natural resonances (CNR) of the targets; 3 ) synthesize E-pulses for the targets and store in a library; 4) generate the impulse response of the target to be discriminated; 5) convolve this impulse response with the E-pulses in the library; and 6) classify the target based on the late-time response of the convolved output.

\section{A. Modeling of the Aircraft}

MiG-29, represented by canonical shapes, is taken as the basic aircraft and its variant has stores (a 
missile) attached under each wing. It has a fuselage of length $\approx 17.3 \mathrm{~m}$ and a wing span of $\approx 11.3 \mathrm{~m} \mathrm{[14].}$ The variant of the MiG-29 (referred as the aircraft with missile) is constructed by adding structural model of a medium range, radar guided, air-to-air R530 missile of length $\approx 3.2 \mathrm{~m}$ [15]. The missile is attached to the wing by four plates. The aircraft is modeled using FEMAP, a commercially available package. The basic aircraft is modeled as 43 surfaces and the aircraft with missile as 63 surfaces. The aircraft surface is then discretized into triangular patches before computing the frequency response. The number of triangular patches is calculated based on the maximum frequency of interest and is described in Section IIIA. Due to the electrically large structure of the target, physical optics (PO) technique is used to generate the frequency response.

\section{B. Computation of Complex Natural Resonances}

The current approaches to determine the complex natural frequencies of arbitrary targets are either based on solving the determinantal equation or by extracting the poles from the impulse response.

1) Solving the Determinantal Equation: It is well known that the singularity expansion method (SEM) is a rigorous method based on extraction of the natural resonances (frequencies) of targets. The scattering process when viewed as a linear time invariant (LTI) system, the natural frequencies represent the poles of the system. The formulation for natural resonance computation using SEM for the case of wire scatterers is presented in [16], [17]. The natural frequencies of the thin wire, denoted by $s_{\alpha}$, are obtained by solving the following polynomial equation [16]

$$
\operatorname{det}\left[Z\left(s_{\alpha}\right)\right]=0 .
$$

Here, $[Z(s)]$ is an $n \times n$ impedance matrix where $n$ represents the number of subdomains on the wire.

Similarly, in the case of complex arbitrary shaped targets, such as cylinder with perturbation, the impedance matrix $[Z(s)]$ can be formed using the electron field integral equation (EFIE) [18]. Note that the formulation in [18] is for $s=j \omega$, i.e., the imaginary axis in the complex plane, and can be modified to cater for the complex frequency $s$ by substituting $j \omega=s / c$. In case of simple targets such as wire scatterers, the root finding algorithms converge to the roots at a faster rate as compared with those of the complex targets. Evidently, the convergence of such algorithms is faster when approximate locations of the roots are known a priori. However, for most of the complex targets, there is little, or no, such prior information. Singaraju et al. [19] have provided a method to obtain an initial guess for the roots of a polynomial using the contour integration method. Using this algorithm as a preliminary procedure, all dominant poles in the complex plane have been located initially by Sun et al. for the case of a regular cylinder [20]; subsequently, other root-finding subroutines such as C05NBF from the NAG library have been used to improve the accuracy of the pole locations. Further, Vechinski has used the same algorithm to compute the poles from a body of revolution (BOR) [21]. However, for all these cases the order or characteristic polynomial is relatively low. For arbitrary complex bodies this technique becomes cumbersome since the polynomial will be several orders higher. Therefore, such an approach is not tractable when the poles of large complex shaped targets are to be obtained. Thus, in this work we adopt the approach to extract the poles from the impulse response of a complex body as described in the following sections.

2) Extracting CNR from Impulse Response: The impulse response can be generated for arbitrary targets using either time domain integral equation (TDIE) [22] or finite difference time domain (FDTD) techniques. Alternatively, the time response can be generated from the frequency response of the scatterers using inverse Fourier transform method. The second approach is used in this work. The frequency response, over the frequency range of interest, is obtained for different targets using appropriate computational electromagnetic techniques. The natural frequencies are then extracted from the impulse response using Prony's method [23].

a) Impulse response from frequency response: There is no single computational electromagnetics (CEM) technique that can effectively cover the entire range of frequencies that is needed for the E-pulse synthesis using high frequency poles. Hence, for low frequency scattering, method of moments (MoM) is used. The low frequency limit is taken to be the frequency at which the characteristic dimension of the body is smaller than $5 \lambda$. This limit has been set by the available supercomputer for this research. At higher frequencies, PO is used.

Essentially, the scattered field obtained from $\mathrm{MoM}$ and $\mathrm{PO}$ at various frequencies are collated to compute the time response of a target excited by a plane wave impulse. However, the frequency response of an ideal impulse function extends over an infinite range of frequencies with a constant amplitude corresponding to an infinite power pulse. Therefore, for practical reasons of limited computer time and storage, such a function cannot be implemented, nor can an infinite power pulse be generated. On the contrary, an approximate smoothed impulse function with a Gaussian temporal variation is well suited for numerical computation even though such a function is effectively time and band limited in nature. Such a function decays rapidly to a negligible value both in time and frequency domains. Hence, a Gaussian pulse is used [22] as the incident waveform.

The impulse response is obtained by taking the inverse Fourier transform of the product of the 
frequency response, obtained using the hybrid CEM technique, and the Fourier transform of the Gaussian pulse. The impulse response obtained for the cylinders are also compared with the results obtained by TDIE [22] for validation of the results.

b) Natural frequencies from impulse response: Evidently, accurate extraction of natural frequencies from a transient response is very important for target recognition. A good method requires to be computationally efficient, automatically operate (i.e., without any initial guess), insensitive to random noise, and estimation of model number. Prony's method meets the first and second requirement and has been used in this paper [24]. A systematic approach based on Prony's method for deriving the CNR, and their residues, is described in [23]. It is well known [25] that Prony's algorithm is sensitive to noise, aliasing, and rank deficiency. Nevertheless, in this work, data generated from computational techniques is without noise and hence the use of Prony's method was deemed sufficient for demonstrating the extraction of natural frequencies for the cases considered. The aliasing problem is overcome by satisfying the Shannon's criteria. However, in practical situations, where the maximal frequency content of the signal is unknown, the technique described by Majda [26] to estimate the sampling rate based on obtaining a minimum condition number is exploited. The rank deficiency problem implies that an a priori knowledge of the number of natural frequencies is required to choose the model order of the system. This choice of number of frequencies is considered to be one of the major issues in the application of the Prony's method and is described in the next subsection. Ilavarasan et al. [27] cautions that at times, estimation of natural resonances (poles of the LTI system) results in positive damping coefficients. It is observed that poles with positive damping coefficients are not obtained if the number of poles is carefully chosen for estimating the natural frequencies.

3) Choice of Number of Poles: Theoretically, a finite sized perfectly conducting scatterer has an infinite number of natural resonances. Nonetheless, the transient response for a body, whether obtained either from experimental measurements or from a numerical solution of the scattering problem, is band limited and, hence, has a limited number of poles. Hence estimation of the number of poles (which is the model order of the system when the scattering process is viewed as a linear time invariant system) is essential in accurate determination of natural resonances.

Researchers from the field of control theory use the Hankel singular values [28] of a system to estimate the model order. In this paper, we use these values to estimate the model order of the system and hence the number of poles in the Prony's method; and verified using that obtained from Majda et al. [26]. The method based on Hankel singular values is as follows:

a) Hankel singular values: Let the transfer function of the simulated frequency response of the scatterers be denoted by $G(s)$. Note that $G(s)$ is unknown, but that $|G(j \omega)|$, the frequency response is available. The impulse response $I(t)$ is obtained from $|G(j \omega)|$ via an inverse fast Fourier transform (IFFT). Subsequently, estimates of the pole locations are obtained from the impulse response $I(t)$ using Prony's method. Let $\hat{G}(s)$ represent the estimate of the transfer function $G(s)$ with these estimated poles. Essentially, in the Hankel norm approximation approach, the Hankel norm of the error $\| G(s)-$ $\hat{G}(s) \|_{H}$ is minimized. In practice, this minimization is carried out as follows. A number $N$ large enough is specified for the Prony's algorithm and an estimate $\hat{G}(s)$ obtained. Subsequently, the Hankel singular values of $\hat{G}(s)$ are computed, and the less significant of these are removed using the algorithm described in [28] to obtain a second estimate $\hat{\hat{G}}(s)$.

The final pole locations (i.e., poles of $\hat{\hat{G}}(s))$ are validated in two different ways. First, these pole locations are used as initial values in the root finding algorithm that solves the determinantal equation (1) in the frequency domain method. Second, the impulse response $\hat{\hat{I}}(t)$ of $\hat{\hat{G}}(s)$ can easily be computed from the poles and residues. This response is then compared with the impulse response $I(t)$ generated originally from the frequency response $|G(j \omega)|$. Here, signals are compared using the following measure "variance accounted for" (VAF) expressed as a percentage:

$$
\operatorname{VAF}(I, \hat{\hat{I}})=\left(1-\frac{\operatorname{variance}(I(t)-\hat{\hat{I}}(t))}{\operatorname{variance}(I(t))}\right) 100 \% .
$$

This measure is used frequently in the model identification and control literature for validation of results. (See [29], for example.) Observe that when the signals are identical, VAF is 100 . As the difference between signals becomes significant, VAF reduces considerably.

\section{Discrimination Algorithm}

In this paper, the discrimination of the basic aircraft from the aircraft with stores is attempted using the E-pulse technique. In this work, the effectiveness of using higher frequency poles in the discrimination between basic aircraft and aircraft with stores is measured by a discrimination number (DN). The DN is defined, in the late time, as the ratio of the energy of the convolved output $\tilde{c}_{e}(t)=\tilde{e}(t)(t)$ to the energy of the convolved output $c_{e}(t)=e(t)(t)$ where $\tilde{e}(t)$ and $e(t)$ are, respectively, the E-pulses of aircraft with stores and basic aircraft. Thus, 


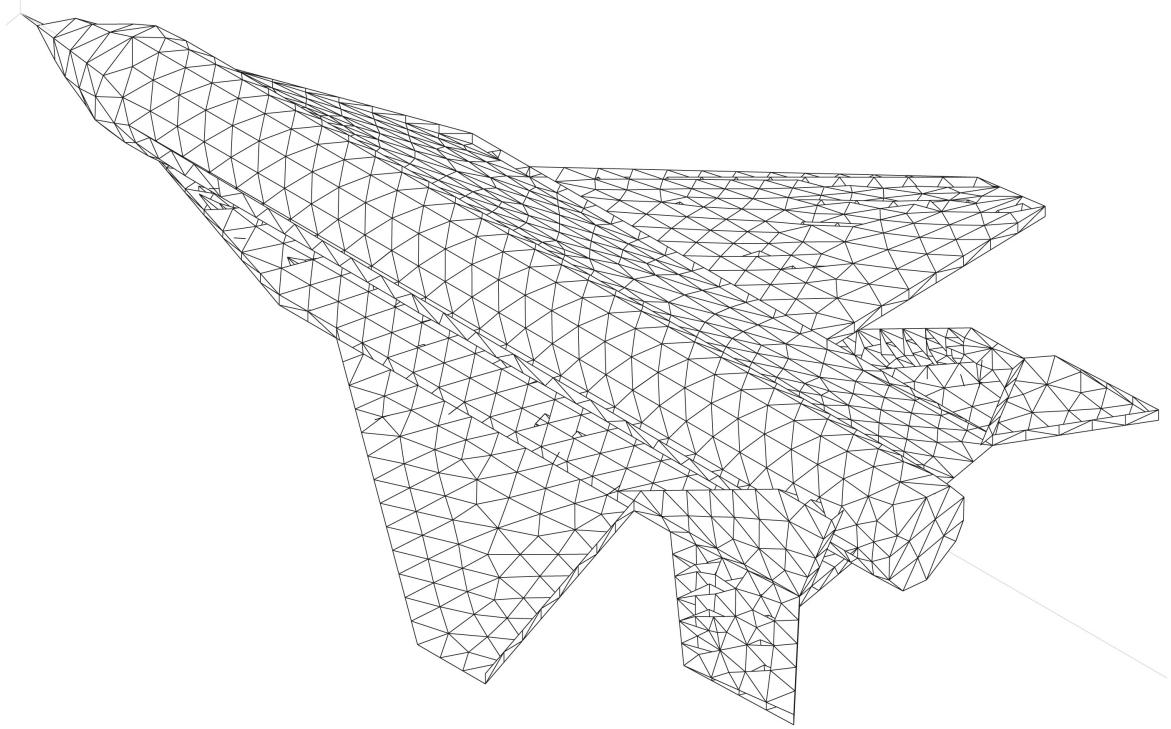

Fig. 1. Triangular patch model of basic aircraft (MiG-29).

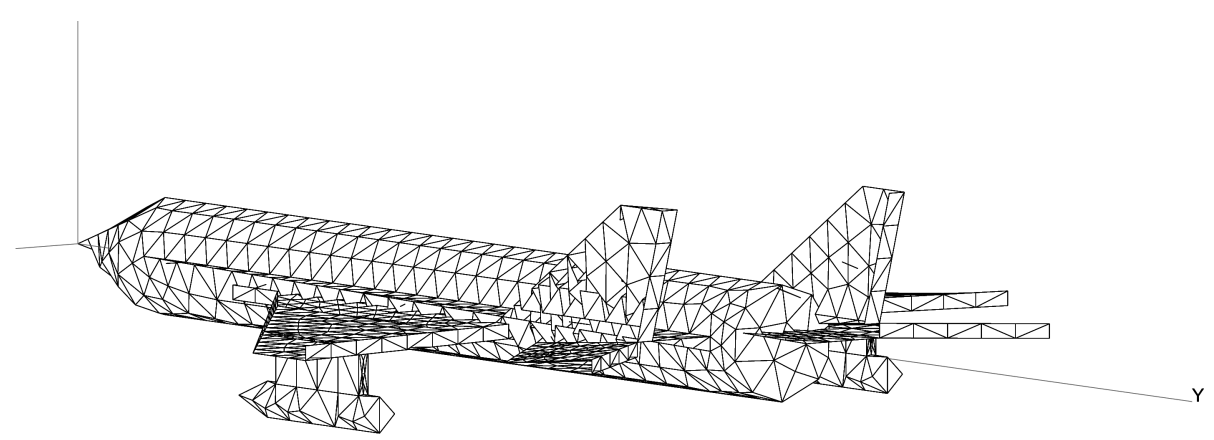

Fig. 2. Triangular patch model of aircraft (MiG-29) with stores.

$$
\mathrm{DN}=10 * \log _{10}\left[\frac{\left[\int_{T_{L}}^{T_{L}+W} \tilde{c}_{e}^{2}(t) d t\right]}{\left[\int_{T_{L}}^{T_{L}+W} c_{e}^{2}(t) d t\right]}\right]
$$

where $W$ is a suitable window for late time, $T_{e}$ is the early time, $T_{L}=T_{p}+2 T_{r}$ is the beginning of the late time, $T_{p}$ is the effective pulse duration used in the measurement system, and $T_{t r}$ is the maximal one-way transit time of the target. Note that with this definition, we get $\mathrm{DN}>0$ for any target other than the target for which the E-pulse is designed.

\section{NUMERICAL RESULTS}

In this section, the discrimination capability of the technique proposed is illustrated for the case of basic aircraft and its minor variation with stores attached.

A. Frequency Response for the MiG-29 Aircraft and its Variant

The minimum resonant frequency for a typical fighter aircraft is reported to be in the range of
5-10 MHz [30] and for the missile is of the order of 50-200 MHz [30]. Hence, in order to characterize the features of both the basic aircraft and the missile, frequency response up to $500 \mathrm{MHz}$ is necessary. Because of the electrically large structure of the aircraft, PO technique is found adequate for computing the frequency response. The PO technique implemented in FEKO requires that the edge of the triangle $l_{e}$ meets the criteria $l_{e}<\lambda / 6$. Using this constraint, the basic aircraft is discretized into 11974 elements and the aircraft with missile is discretized into 48585 elements. Figs. 1 and 2 show the triangular patch model of the basic aircraft and the aircraft with stores, respectively.

The frequency response of the basic aircraft and the aircraft with missile for an incidence for $\theta=90^{\circ}$ and $\phi=0^{\circ}$ is shown in Fig. 3. It can be seen from the figure that the frequency response is high at $17 \mathrm{MHz}$ corresponding to the fuselage dimension. It is clearly seen from the figure that there is a peak in the frequency response of the aircraft with missile case at a frequency of $\approx 100 \mathrm{MHz}$ corresponding to the fuselage of the missile. Further, a peak is seen for the case of the aircraft with missile at $\approx 150 \mathrm{MHz}$ which 


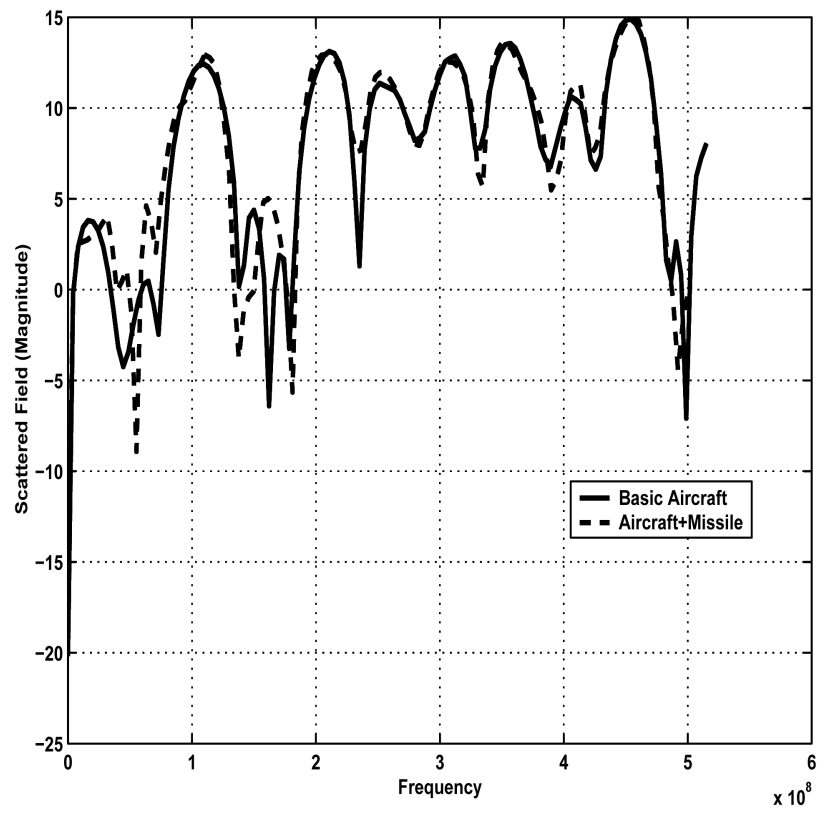

Fig. 3. Frequency response for aircraft and its variant.

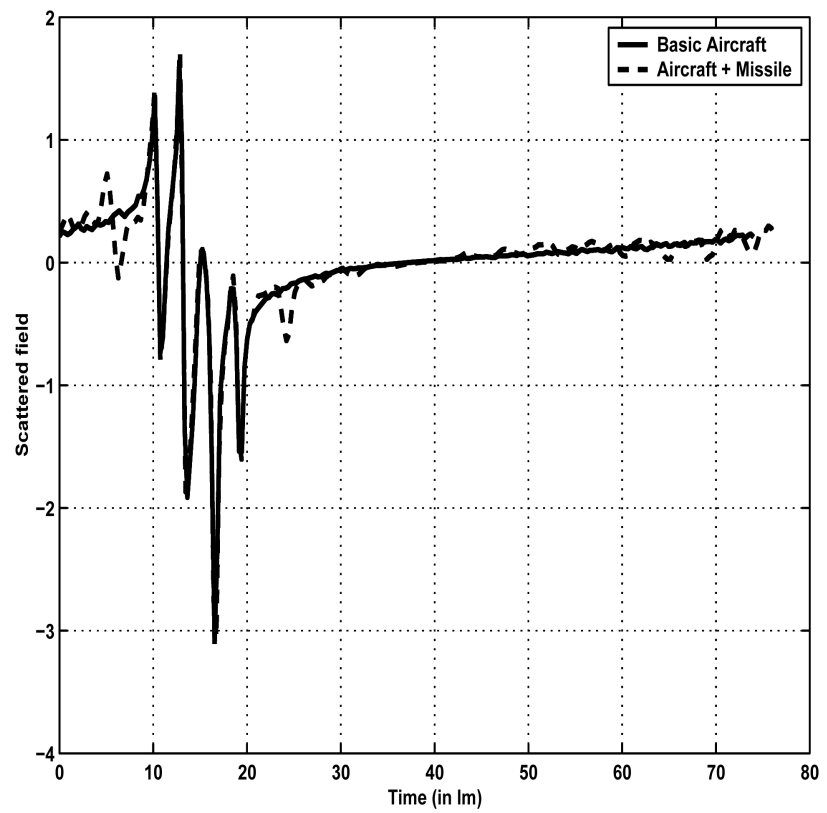

Fig. 4. Impulse response for aircraft and its variant.

corresponds to one of the four plates used to attach the missile with the wing.

\section{B. Impulse Response of the MiG-29 Aircraft and its Variant}

The impulse response is generated for an incident Gaussian pulse of pulsewidth $T=1.2 \mathrm{~lm}$, where $\mathrm{lm}$ represents light-meter. The impulse response for both the aircraft and its variant is given in Fig. 4. The effect of the inclusion of the missile with the aircraft is clearly seen in the impulse response. The deviations in the impulse response for the aircraft with

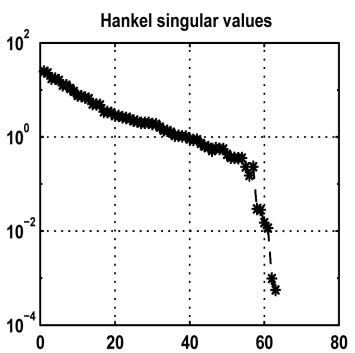

(a)

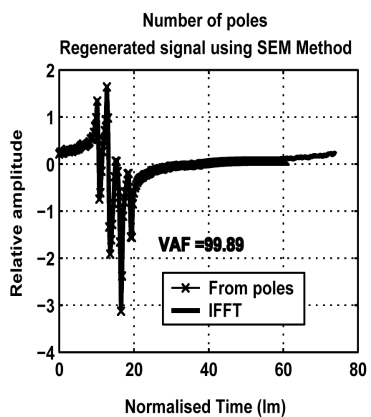

(c)

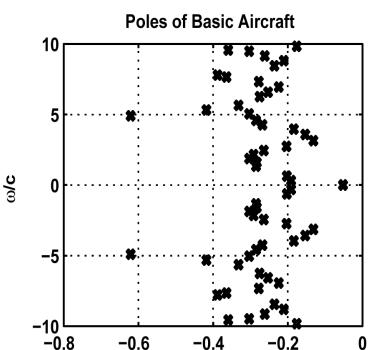

(b)

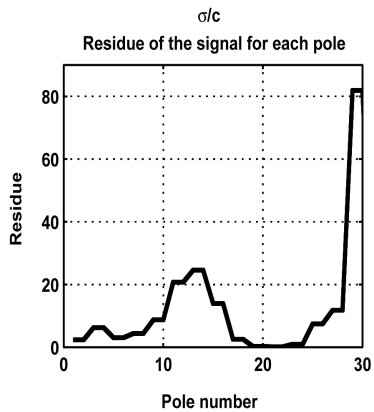

(d)
Fig. 5. Basic aircraft. (a) Hankel singular values. (b) Poles. (c) Impulse response (regenerated using SEM). (d) Residues.

missile case compared with that of the basic aircraft, correspond to the location of the stores.

\section{Complex Natural Resonances for Aircraft}

The natural frequencies for both the aircraft are extracted from the impulse response using the Prony's method. Fig. 5 shows the Hankel singular values, the poles and residues and the validity of the poles by comparing the impulse response generated from the poles using the SEM method, and the computed impulse response. The VAF measure is found to be $99.89 \%$ for this case. Similar graph for the aircraft with missile is given in Fig. 6 and the VAF for this case is found to be $98.6 \%$. Fig. 7 shows the poles of both the aircraft. It is observed from the graph that the effect of the overlaying of the structure due to the cylindrical part of the fuselage and the plate part due to the wing is clearly seen on the poles.

\section{Discrimination Between the Targets}

The discrimination problem is attempted between the two aircraft using the E-pulse technique. The E-pulses are synthesized for the basic aircraft and aircraft with stores. It is observed that for the canonical shaped bodies, the value of the duration $\left(T_{e}\right)$ of the E-pulse is a function of the maximum frequency component among the modes and $T_{e}$ is chosen such that the minimum $T_{e}$ is greater than [10]:

$$
T_{e}=2 N \frac{\pi}{\omega_{\max }} .
$$




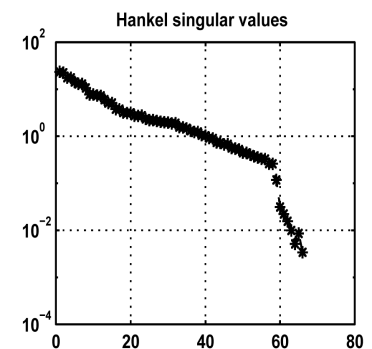

(a)

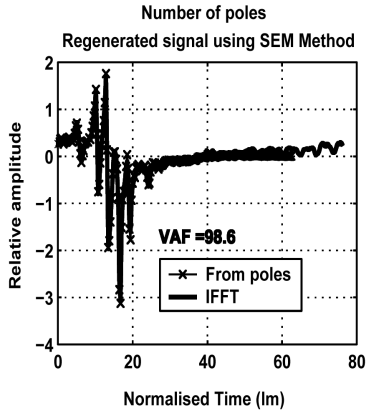

(c)

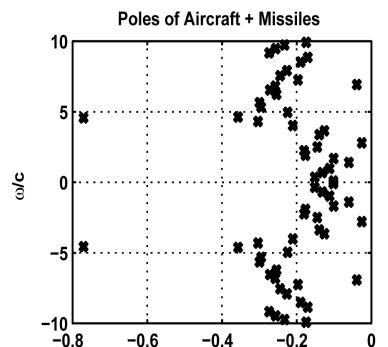

(b)

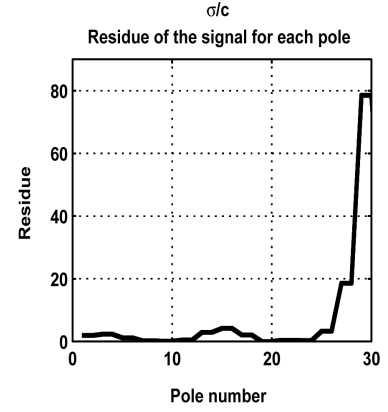

(d)
Fig. 6. Aircraft with Missile. (a) Hankel singular values. (b) Poles. (c) Impulse response (regenerated using SEM). (d) Residues.

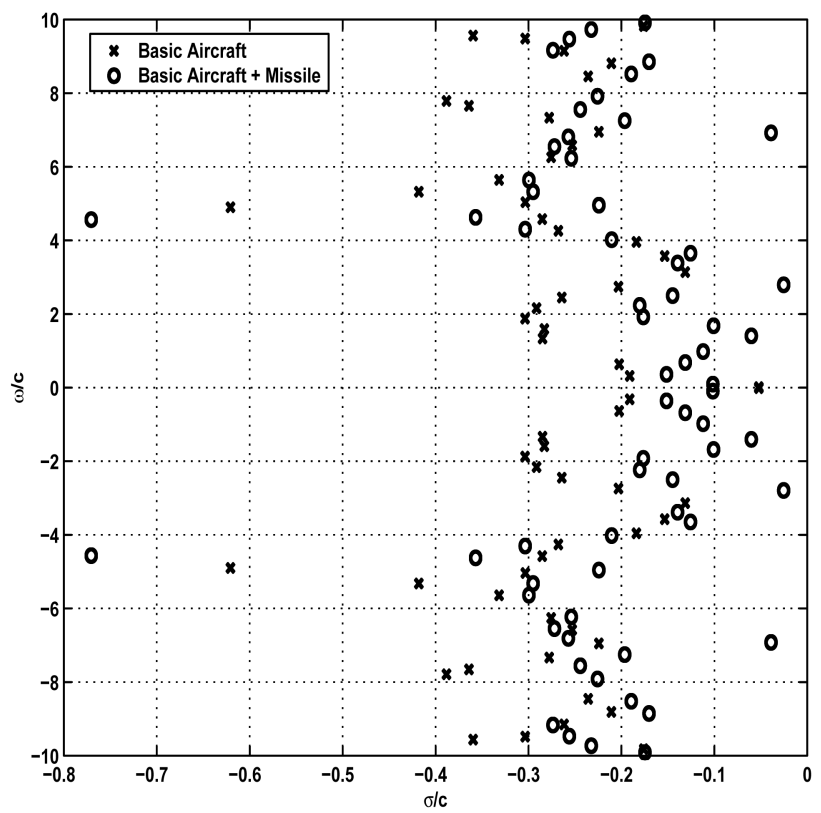

Fig. 7. Poles of aircraft.

For complex bodies such as aircraft, slight variations are observed when the value of $T_{e}$ is altered.

Fig. 8 shows the E-pulses for the basic aircraft for three values of $T_{e}$ and the variation in the E-pulse construction can be seen from the figure.

\section{E. Convolution Output of the Response of Basic Aircraft with the E-pulse}

The convolution output, of the response of basic aircraft with the E-pulses of different duration given

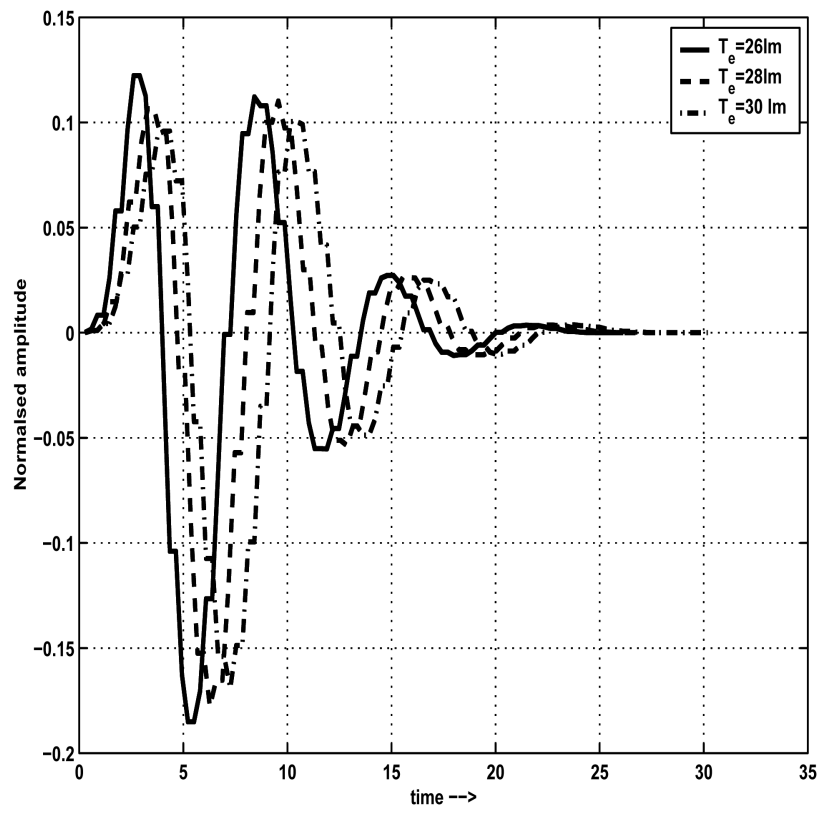

Fig. 8. E-pulses for basic aircraft with varying $T_{e}$.

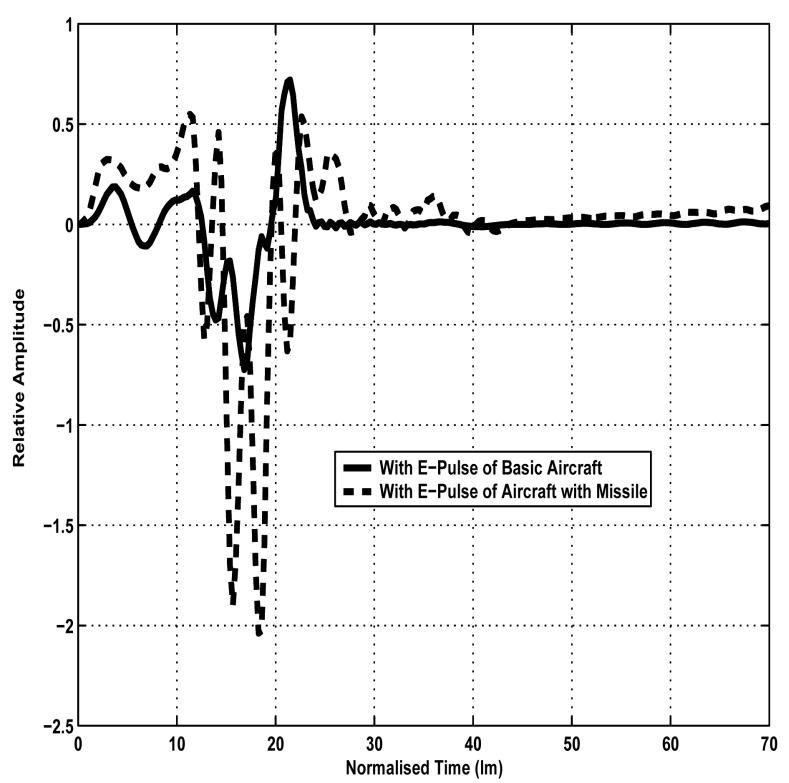

Fig. 9. Convolution output of response of basic aircraft with E-pulse with $T_{e}=26 \mathrm{~lm}$. (a) Basic aircraft. (b) Aircraft with missile.

in Fig. 8, is shown in Figs. 9 and 10. The convolution output in the late time is found to be minimum for the basic aircraft in all these cases. This implies that the basic aircraft is discriminated against the aircraft with missile case successfully.

Fig. 11 shows the discrimination number for the response of the basic aircraft with the E-pulses of the aircraft with missile for different values of $T_{e}$. The discrimination capability is high beyond a frequency of $100 \mathrm{MHz}$. To summarize, these figures effectively demonstrates the capability to discriminate an aircraft with missile against the basic aircraft. 


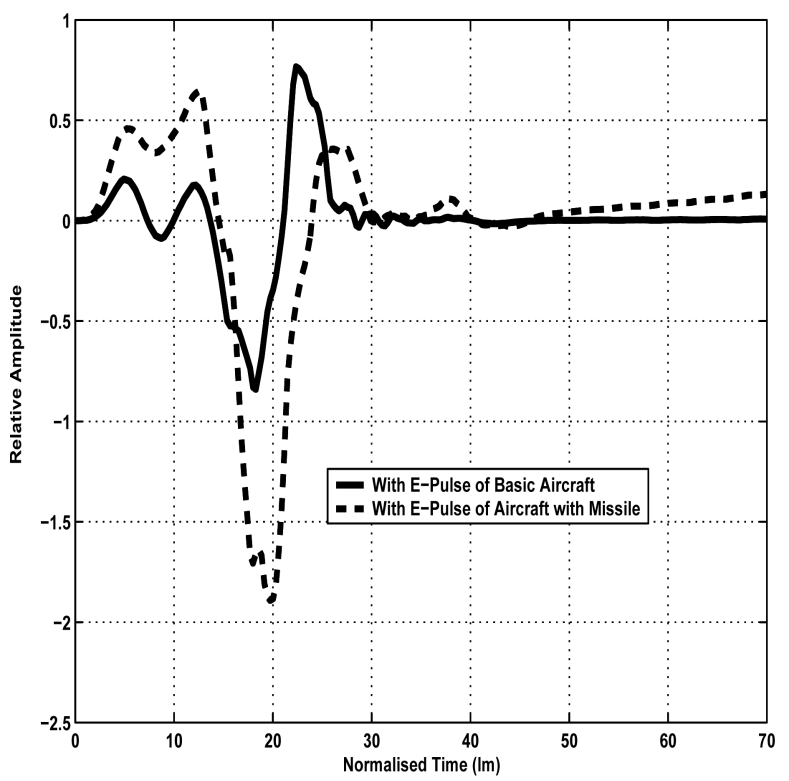

Fig. 10. Convolution output of response of basic aircraft with E-pulse with $T_{e}=30 \mathrm{~lm}$. (a) Basic aircraft. (b) Aircraft with missile.

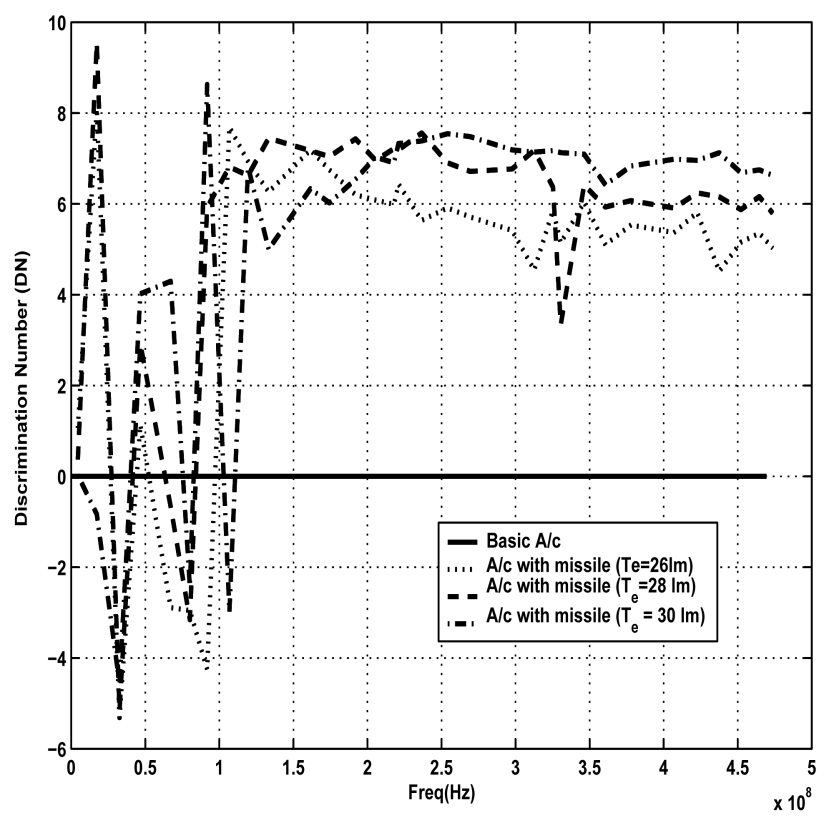

Fig. 11. DN for basic aircraft with varying $T_{e}$.

\section{CONCLUSION}

In this paper, the discrimination capability of the E-pulse technique for discriminating between full-scale model of an aircraft, represented by canonically shaped bodies is demonstrated.

Specifically, the discrimination between two aircraft of the same class with structural modifications is carried out. It is shown that it is possible to discriminate the response from the basic aircraft (MiG-29) against its variant which is another MiG-29 carrying stores under the wing. It is shown that the $\mathrm{DN}$ increases to $6 \mathrm{~dB}$ when the poles of frequencies above $140 \mathrm{MHz}$ are considered in the synthesis of E-pulse.

\author{
K. RAJALAKSHMI MENON \\ Centre for Airborne Systems \\ Min. of Defence (R\&D) \\ 80 Yemalur \\ Bangalore 560037 \\ India \\ E-mail: (raj@cabsdrdo.in)
}

N. BALAKRISHNAN

Dept. of Aerospace Engineering Indian Institute of Science

Bangalore 560012

India

K. RAMCHAND

Centre for Airborne Systems

Min. of Defence (R\&D)

80 Yemalur

Bangalore 560037

India

\section{REFERENCES}

[1] Nebabin, V. G.

Methods and Techniques of Radar Recognition. Norwood, MA: Artech House, 1995.

[2] Zyweck, A., and Bogner, R. E.

Radar target classification of commercial aircraft. IEEE Transactions on Aerospace and Electronic Systems, 32 (Apr. 1996), 598-606.

[3] Williams, R., and Westerkamp, J.

Automatic target recognition of time critical moving target using $1 \mathrm{~d}$ high range resolution radar.

IEEE AES Systems Magazine, (Apr. 2000), 2455-2462.

[4] Moffat, D. L., and Mains, R. K.

Detection and discrimination of radar targets. IEEE Transactions on Antenna and Propagation, 23 (May 1975), 358-367.

[5] Berni, A. J.

Target identification by natural resonance estimation. IEEE Transactions on Aerospace and Electronic systems, 11 (Mar. 1975), 147-154.

[6] Rothwell, E., Nyquist, D. P., Chen, K.-M., and Drachman, B.

Radar target discrimination using the extinction-pulse technique.

IEEE Transactions on Antennas and Propagation, 33 (Sept. 1985), 929-937.

[7] Lin, C. A., and Cordaro, J. T.

Determination of the SEM parameters for an aircraft model from the transient surface current. Electromagnetics, 3 (Mar. 1983), 65-75.

[8] Mooney, J. E., Ding, Z., and Riggs, L. S.

Robust target identification in white Gaussian noise for ultra wide band systems.

IEEE Transactions on Antennas and Propagation, 46 (Dec. 1998), 1817-1823.

[9] Li, Q., Ilavarasan, P., Ross, J. E., Rothwell, E. J., Chen,

K.-M., and Nyquist, D. P.

Radar target identification using a combined early-time/late-time e-pulse technique.

IEEE Transactions on Antennas and Propagation, 46 (Sept. 1998), 1272-1277.

[10] Baum, C. E., Rothwell, E. J., Chen, K.-M., and Nyquist, D. P.

The singularity expansion method and its application to target identification.

Proceedings of the IEEE, 79 (Oct. 1991), 1481-1492. 
[11] Lee, J.-H., Choi, I.-S., and Kim, H.-T.

Natural frequency-based neural network approach to radar target recognition.

IEEE Transactions on Signal Processing, 51 (Dec. 2003), 3191-3197.

[12] Lee, J.-H., and Kim, H.-T.

Radar target recognition based on late time representation: Closed-form expression for criterion. IEEE Transactions on Antennas and Propagation, 54 (Sept. 2006), 2455-2462.

[13] Ksienski, A. A., Lin, Y.-T., and White, L. J.

Low-frequency approach to target identification.

Proceedings of the IEEE, 63 (Dec. 1975), 1651-1660.

[14] Janes all the world's aircraft.

James Information Group, 1340 Braddock Place, Suite 300, Alexandria, VA 22313.

[15] Janes Weapon Systems.

James Information Group, 1340 Braddock Place, Suite 300, Alexandria, VA 22313.

[16] Tesche, F. M.

On the analysis of scattering and antenna problems using the singularity expansion technique.

IEEE Transactions on Antennas and Propagation, 21 (Jan. 1973), 53-62.

[17] Menon, K. R., Balakrishnan, N., Rao, S. M., and

Ramchand, K.

A simple technique for discrimination between radar targets with minor variations.

Microwave and Optical Technology Letters, 28, 5 (Mar. 2001), 336-342.

[18] Rao, S. M., Wilton, D. R., and Glisson, A. W. Electromagnetic scattering by surfaces of arbitrary shape. IEEE Transactions on Antennas and Propagation, 30 (May 1982), 409-418.

[19] Singaraju, B. K., Giri, D. R., and Baum, C. E. Further developments in the application of contour integration to evaluation of the zeros of analytic functions and relevant computer programs.

Mar. 1976, aFWL Math Notes 42.

[20] Sun, W., Chen, K.-M., Nyquist, D. P., and Rothwell, E. J. Determination of the natural modes for a rectangular plate.

IEEE Transactions on Antennas and Propagation, 38 (May 1990), 643-652.
[21] Vechinski, S. R.

Natural resonances of bodies of revolution.

Ph.D. dissertation, Auburn University, Auburn, AL, 1989.

[22] Rao, S. M. (Ed.)

Time Domain Electromagnetics.

New York: Academic Press, 1999.

[23] Van Blaricum, M. L., and Mittra, R.

A technique for extracting the poles and residues of a system directly from its transient response.

IEEE Transactions on Antennas and Propagation, 23 (Nov. 1975), 777-781.

[24] Choi, I.-S., Lee, J.-H., Kim, H.-T., and Rothwell, E.

Natural frequency extraction using late-time evolutionary programming-based CLEAN.

IEEE Transactions on Antennas and Propagation, 51 (Dec. 2003), 3285-3292.

[25] Poggio, A. J., Van Blaricum, M. L., and Miller, E. K. Evaluation of a processing technique for transient data. IEEE Transactions on Antennas and propagation, 26 (Jan. 1978), 165-173.

[26] Majda, G., Strauss, W. A,. and Wei, M.

Computations of exponentials in transient data.

IEEE Transactions on Antennas and Propagation, 37 (Oct. 1989), 1284-1290.

[27] Ilavarasan, P., Rothwell, E. J., Chen, K.-M., and Nyquist, D. P.

Natural resonance extraction from multiple data sets using a genetic algorithm.

IEEE Transactions on Antennas and Propagation, 43 (Aug. 1995), 900-904.

[28] George, K.

$\mathcal{H}_{\infty}$-based robust controller for aerospace vehicles. Ph.D. dissertation, Indian Institute of Science, Bangalore, India, 1996.

[29] George, K., Verhaegen, M., and Scherpen, J. M. A.

A systematic and numerically efficient procedure for stable dynamic model inversion of LTI systems.

Presented at the 38th IEEE Conference on Decision and Control, Phoenix, AZ, Dec. 1999.

[30] Taylor, J. (Ed.)

Introduction to Ultra-WideBand Radar Systems.

Boca Raton, FL: CRC Press, 1995. 